\title{
Psoriatic Arthritis Diagnosis and Management in the Era of Telehealth
}

\author{
George Han, MD, PhD
}

W ith the rise of telehealth utilization during the COVID-19 pandemic, clinical care delivery has undergone a substantial shift. This is especially true in dermatology, as utilization of telehealth has jumped from under $15 \%$ to more than $95 \%$ of dermatologists after the COVID-19 pandemic. ${ }^{1}$ However, with this new form of care delivery, it is important to ensure that patients don't get left behind, either due to socioeconomic/ language barriers $^{2}$ or hesitancy about the conditions being treated.

It may not be surprising to know that the idea of using telemedicine for rheumatology is not new. Indeed, a report from 20 years ago outlined the high level of both satisfaction with live interactive telehealth visits for rheumatologic conditions and diagnostic accuracy as compared to in-person visits. ${ }^{3}$ Through guided palpation and careful history taking, it is possible to conduct a thorough visit and even manage biologics, diagnose active arthritis/enthesitis via photographs, and evaluate pain through a visual analog scale. ${ }^{4}$ As far as dermatology is concerned, it is clear that certain situations seem to be better suited for teledermatology, such as follow-up visits for acne/rosacea. ${ }^{1}$ But what of psoriatic arthritis (PsA)? Does telehealth have the potential to mitigate our undertreatment of this important condition, which finds about half of patients being treated with only topical therapy or no treatment at all? ${ }^{5}$ Or can we modulate our visits to accommodate these patients, taking care of not only their visible psoriasis but also the underlying PsA?

Psoriasis is well suited for teledermatology management in general, especially once the diagnosis is made. Multiple studies have shown diagnostic equivalence with in-person care and even similar outcomes after treatment. ${ }^{6,7}$ However, most studies have looked at telemedicine primarily for cutaneous psoriasis, and translating this to screening for and management of PsA is paramount. After all, a delay of only 6 months in diagnosing and treating PsA has been associated with poor outcomes. ${ }^{8}$ Thankfully, we do have some tools that can help. There are 3 validated screening tools for PsA: the Psoriasis Epidemiology Screening Tool (PEST), the Psoriatic Arthritis Screening and Evaluation (PASE), and the Toronto Psoriatic Arthritis Screen (ToPAS) questionnaire. ${ }^{9}$ Of these, the PEST seems to be a reasonable option that is quick and easily deployed; it has shown strong performance in terms of sensitivity, specificity, and negative predictive value/positive predictive value when compared to similar screening tools. ${ }^{10}$ It also should be facile to direct patients to complete the screening tool, as an online version is available on the National Psoriasis Foundation's website (https://www.psoriasis .org/psoriatic-arthritis-screening-test/) where patients can be directed to answer 5 simple questions and report back the outcome. For treatment decisions, this tool also can be used to help identify patients who are good candidates for systemic or biologic therapy or those who should see a rheumatologist. Of course, an in-depth discussion of joint pain, morning stiffness, and tender/ swollen joints may be more fruitful but also more challenging to conduct. I would propose that this can be pared down to a more direct conversation about finger pain/tenderness, tenderness at the elbow/knee (lateral epicondyle/medial femoral condyle), or heel (Achilles) as more common sites of enthesitis, and questioning about back pain or stiffness that improves with movement. ${ }^{9}$

From the Department of Dermatology, Zucker School of Medicine at Hofstra/Northwell, New Hyde Park, New York. Dr. Han is or has been an investigator, consultant/advisor, or speaker for AbbVie, Athenex, Boehringer Ingelheim, Bond Avillion, Bristol-Myers Squibb, Celgene Corporation, Dermavant, Eli Lilly, Janssen, LEO Pharma, MC2, Novartis, Ortho Dermatologics, PellePharm, Pfizer, Regeneron, Sanofi/Genzyme, SUN Pharmaceutical, and UCB.

Correspondence: George Han, MD, PhD (georgehanmdphd@gmail.com).

doi:10.12788/cutis.0313 
By combining the screening tool with these pointed questions, even via telehealth, we can greatly improve our yield in diagnosing PsA while only adding a minute or two to our visits. I'd argue that this is much more fruitful than asking the patient to contort their bodies and camera to show an obscure lesion!

It is interesting to consider areas in dermatology where we might make a notable impact on mortality and morbidity by expanding access to care. Earlier diagnosis of melanoma, for instance, certainly would be in consideration, especially in areas of the country where access to dermatologic care is challenging. Better management of PsA has to be up there on the list of conditions where we immediately can make a tangible difference; we have the tools to do so and excellent therapeutics that are safe and effective. Our colleagues in rheumatology have embraced telemedicine with a "how, not if" approach to embracing new technology, ${ }^{11}$ and it is about time that dermatology takes a similar attitude. The gap between access to dermatologic care in urban areas vs either nonmetropolitan or rural areas is increasing, and dermatology tends to be much more available in well-resourced, urban areas. ${ }^{12}$ There are patients who need our expertise, and if it takes the compromise of adopting a technology that sometimes gives us headaches (we've all been on video visits with a choppy signal and inadequate lighting), we still should try to figure out the best way to do it because it's the right thing to do for these patients. If we don't, the determination of how to conduct teledermatology care will be taken away from us and either insurance companies or corporations not guided by dermatologists may try to enter this health care void and decide how to provide these services.

\section{REFERENCES}

1. Kennedy J, Arey S, Hopkins Z, et al. Dermatologist perceptions of teledermatology implementation and future use after COVID-19: demographics, barriers, and insights. JAMA Dermatol. 2021;157:595-597.

2. Rodriguez JA, Saadi A, Schwamm LH, et al. Disparities in telehealth use among California patients with limited English proficiency. Health Aff (Millwood). 2021;40:487-495.

3. Leggett P, Graham L, Steele K, et al. Telerheumatology-diagnostic accuracy and acceptability to patient, specialist, and general practitioner. Br J Gen Pract. 2001;51:746-748.

4. Costa L, Tasso M, Scotti N, et al. Telerheumatology in COVID-19 era: a study from a psoriatic arthritis cohort [published online June 11, 2020]. Ann Rheum Dis. doi:10.1136/annrheumdis-2020-217806

5. Lebwohl MG, Bachelez H, Barker J, et al. Patient perspectives in the management of psoriasis: results from the population-based Multinational Assessment of Psoriasis and Psoriatic Arthritis Survey. J Am Acad Dermatol. 2014;70:871-881; E871-E830.

6. Armstrong AW, Chambers CJ, Maverakis E, et al. Effectiveness of online vs in-person care for adults with psoriasis: a randomized clinical trial. JAMA Netw Open. 2018;1:E183062.

7. Koller S, Hofmann-Wellenhof R, Hayn D, et al. Teledermatological monitoring of psoriasis patients on biologic therapy. Acta Derm Venereol. 2011;91:680-685.

8. Haroon M, Gallagher P, FitzGerald O. Diagnostic delay of more than 6 months contributes to poor radiographic and functional outcome in psoriatic arthritis. Ann Rheum Dis. 2015;74:1045-1050.

9. Gottlieb A, Merola JF. Psoriatic arthritis for dermatologists. JDermatolog Treat. 2020;31:662-679.

10. Urruticoechea-Arana A, Benavent D, Leon F, et al. Psoriatic arthritis screening: a systematic literature review and experts' recommendations. PLoS One. 2021;16:E0248571.

11. Bateman J, Cleaton N. Managing patients using telerheumatology: lessons from a pandemic. Best Pract Res Clin Rheumatol. 2021; 35:101662.

12. Feng H, Berk-Krauss J, Feng PW, et al. Comparison of dermatologist density between urban and rural counties in the United States. JAMA Dermatol. 2018;154:1265-1271. 